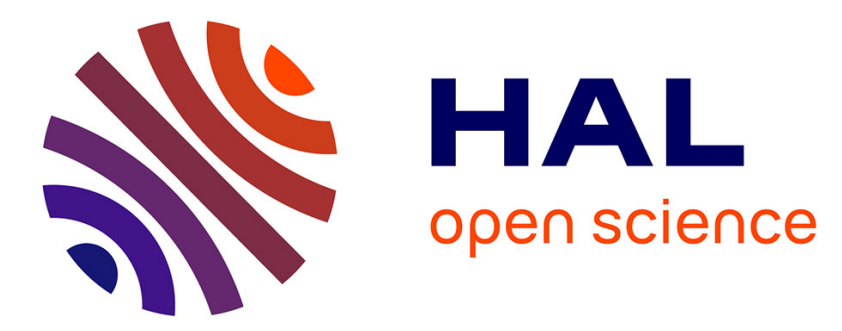

\title{
Mapping between atomistic simulations and Eshelby inclusions in the shear deformation of an amorphous silicon model
}

\author{
Tristan Albaret, Anne Tanguy, Francesca Boioli, David Rodney
}

\section{To cite this version:}

Tristan Albaret, Anne Tanguy, Francesca Boioli, David Rodney. Mapping between atomistic simulations and Eshelby inclusions in the shear deformation of an amorphous silicon model. Physical Review E , 2016, 93 (5), pp.053002. 10.1103/PhysRevE.93.053002 . hal-02304939

\section{HAL Id: hal-02304939 \\ https://univ-lyon1.hal.science/hal-02304939}

Submitted on 14 Jul 2020

HAL is a multi-disciplinary open access archive for the deposit and dissemination of scientific research documents, whether they are published or not. The documents may come from teaching and research institutions in France or abroad, or from public or private research centers.
L'archive ouverte pluridisciplinaire HAL, est destinée au dépôt et à la diffusion de documents scientifiques de niveau recherche, publiés ou non, émanant des établissements d'enseignement et de recherche français ou étrangers, des laboratoires publics ou privés. 


\title{
Mapping between atomistic simulations and Eshelby inclusions in the shear deformation of an amorphous silicon model
}

\author{
T. Albaret, ${ }^{1}$ A. Tanguy, ${ }^{1,2}$ F. Boioli, ${ }^{1}$ and D. Rodney ${ }^{1}$ \\ ${ }^{1}$ Institut Lumière Matière, UMR5306 Université Lyon 1-CNRS, Université de Lyon, F-69622 Villeurbanne Cedex, France \\ ${ }^{2}$ LaMCos, INSA-Lyon, CNRS UMR5259, Université de Lyon, F-69621 France
}

(Received 7 July 2015; revised manuscript received 9 February 2016; published 5 May 2016)

\begin{abstract}
In this paper we perform quasistatic shear simulations of model amorphous silicon bulk samples with Stillinger-Weber-type potentials. Local plastic rearrangements identified based on local energy variations are fitted through their displacement fields on collections of Eshelby spherical inclusions, allowing determination of their transformation strain tensors. The latter are then used to quantitatively reproduce atomistic stress-strain curves, in terms of both shear and pressure components. We demonstrate that our methodology is able to capture the plastic behavior predicted by different Stillinger-Weber potentials, in particular, their different shear tension coupling. These calculations justify the decomposition of plasticity into shear transformations used so far in mesoscale models and provide atomic-scale parameters that can be used to limit the empiricism needed in such models up to now.
\end{abstract}

DOI: 10.1103/PhysRevE.93.053002

\section{INTRODUCTION}

The Eshelby inclusion [1] is the cornerstone of most mesoscopic models of plasticity and rheology in amorphous materials [2-11]. Eshelby inclusions can be thought of as localized plastic cores embedded in an elastic homogeneous surrounding. Their relevance to amorphous plasticity is rooted in the early concept introduced by Argon [12] and Spaepen [13], who proposed that elementary deformation units in sheared amorphous systems consist in local irreversible rearrangements involving few tens of particles. The latter were initially related to the free volume theory [13] and called shear transformations [12] but are now commonly called shear transformation zones (STZs) after the work of Langer et al., who, among others, extended the STZ concept to construct mean-field constitutive models of the mechanics and rheology of amorphous solids [14-16]. STZs have been evidenced experimentally through confocal microscopy in colloidal glasses $[17,18]$ and their signature in displacement fields has been observed for decades in atomistic simulations [11,15,19-22]. However, the respective roles of shear and densification are still a matter of debate that should be solved to propose a realistic, and, if necessary, material-dependent, model of plasticity in amorphous materials.

From a continuum perspective, a strain transformation in a localized volume embedded in a homogeneous solid leads to a characteristic elastic response of the material, which depends on the elastic constants, the volume and shape of the inclusion, and the boundary conditions. Determining the strain and stress fields is the so-called inclusion problem, first addressed by Eshelby [1]. When the local transformation is a shear strain, the material response has a quadrupolar symmetry, as commonly reported in atomistic simulations [11,15,19-22]. Using the analytic solution for the cylindrical inclusion problem in two dimensions, it was shown that the energy minimum for a collection of pure shear inclusions is obtained by a linear arrangement that can be associated with the formation of a shear band [8]. However, the nucleation of such shear inclusions and the associated energy barriers are still open questions [23].
Despite their ubiquitous nature, few attempts have been made to extract the features of Eshelby inclusions from molecular dynamics (MD) simulations in amorphous systems [21,22]. As a result, mesoscopic models have so far relied on rough estimates of both the size and the strain of the Eshelby inclusions [2,5-9,11,24,25]. Moreover, it has never been verified from the atomistic scale if the stress-strain curve of a sheared amorphous solid could be reproduced solely from the knowledge of the underlying collection of Eshelby inclusions. This question is far from trivial because amorphous solids are known to be elastically heterogeneous [26] and it is therefore not clear whether a homogeneous model, which is only a first-order approximation, can faithfully reproduce atomistic data. Such a connection would, however, be highly beneficial to multiscale approaches of plasticity [3].

In this paper, we perform in three dimensions constantvolume quasistatic shear atomistic simulations of model amorphous silicon bulk samples described by two empirical interatomic potentials that differ in the strength of their bond directionality. Plastic rearrangements are identified following a technique based on local energy differences [27]. The corresponding atomistic displacement fields are then fitted to a superposition of spherical Eshelby inclusions to determine their transformation tensors. The latter are finally used to predict the stress-strain relation, which is compared to the atomistic result. The accuracies of different fitting schemes are compared, emphasizing the respective role of local shear and local densification on the macroscopic constitutive law. Also, the comparison between different interatomic potentials allows us to underline the consequences of specific chemical bondings for local shear and densification.

\section{MOLECULAR DYNAMICS SIMULATIONS AND METHOD OF ANALYSIS}

\section{A. Preparation and deformation of the amorphous samples}

Amorphous samples were obtained using a protocol similar to that in Ref. [27]. Most of the samples contained $N=32768$ atoms in cubic periodic cells with a linear dimension of 
TABLE I. Structural and elastic properties of SW and SWM initial amorphous samples compared to experimental data. $Z_{\text {av }}$ is the average coordination number (cutoff, $2.85 \AA$ ); $G$ is the shear modulus, and $v$ Poisson's ratio.

\begin{tabular}{lcccc}
\hline \hline & Density $\left(\mathrm{g} / \mathrm{cm}^{3}\right)$ & $Z_{\mathrm{av}}$ & $G(\mathrm{GPa})$ & $v$ \\
\hline SW & 2.30 & 4.07 & 35 & 0.34 \\
SWM & 2.20 & 3.98 & 60 & 0.25 \\
Expt. & $2.22-2.28^{\mathrm{a}}$ & $3.6-3.9^{\mathrm{a}}$ & $33-49^{\mathrm{b}}$ & $0.22-0.27^{\mathrm{b}}$ \\
\hline \hline
\end{tabular}

${ }^{\mathrm{a} F r o m ~ R e f s . ~[31] ~ a n d ~[32] . ~}$

${ }^{\mathrm{b}}$ From Refs. [33] and [34].

about $8.7 \mathrm{~nm}$. To test size effects, a few runs were performed on samples with $N=262144$ atoms with linear dimensions twice as large as the reference configuration. We started from systems equilibrated in the liquid phase at $3500 \mathrm{~K}$ with Tersoff potential [28]. The samples were quenched at constant volume with a quench rate of $10^{11} \mathrm{~K} / \mathrm{s}$ down to about $100 \mathrm{~K}$. The potential energy was then minimized using a damped MD algorithm until all the atomic forces were below $10^{-3} \mathrm{eV} / \AA$. Samples were further relaxed at $100 \mathrm{~K}$ during 20 ps using two parametrizations of the Stillinger-Weber potential: the original parametrization of Stillinger and Weber (SW) [29] and a modification of SW to model brittle fracture (SWM) [30]. These two potentials display different plastic behaviors in crystalline silicon: SW samples are more ductile, while SWM samples are more brittle [30,42]. Practically, the SWM parametrization shows a higher bond directionality, with a three-body term twice as large as the original SW potential.

Before applying the deformation, we relaxed both the atomic positions using the above force criterion and the cell linear dimensions to obtain equilibrium configurations with a pressure below $0.5 \mathrm{MPa}$ in absolute value. This preparation protocol leads to initial amorphous structures with properties summarized in Table I, in fair agreement with experiments.

The samples were then subjected to series of simple shear deformations with an engineering shear strain increment $\delta \gamma_{x y}=10^{-3}$. The strain increments are applied to the cell vectors to ensure a homogeneous $(x y)$ shear deformation in the periodic box, leaving the cell vector along $z$ unchanged. A potential energy minimization using damped MD follows each increment, with the same force criterion as above. The output of the atomistic simulations is thus a succession of equilibrium configurations at increasing strains, $\gamma_{i}=i \delta \gamma_{x y}$.

Figure 1 displays representative examples of stress-strain curves obtained with both versions of the SW potential. At small strains, the curves are close to linear, while an increasing plastic activity is progressively evidenced by a growing negative curvature at intermediate strains. At around $20 \%$ strain, the curves flatten into a plateau, which ends up with a large stress drop that corresponds to the formation of a shear band aligned with the direction of simple shear $(y)$. After this, the system enters the plastic flow regime.

\section{B. Identification of plastic events}

In order to identify plastic events, we computed after each forward strain increment (i.e., with a positive $\delta \gamma_{x y}$ ) the reverse step by applying a $-\delta \gamma_{x y}$ deformation and relaxing the system again. For each strain $\gamma_{i}$, we thus have a current

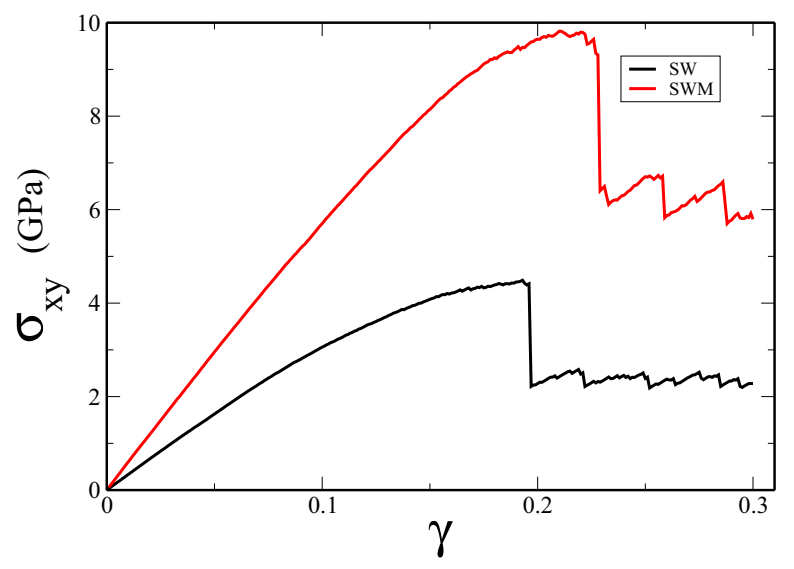

FIG. 1. Stress-strain curves obtained at constant volume under quasistatic simple shear. Black curve: SW potential. Red curve: SWM potential.

configuration, $\{\boldsymbol{r}\}^{\text {curr }}$, and a "reversed" configuration, $\{\boldsymbol{r}\}^{\text {rev }}$, obtained after an incremental strain cycle $\gamma_{i} \rightarrow \gamma_{i+1} \rightarrow \gamma_{i}$. If no plastic event occurs between $\gamma_{i}$ and $\gamma_{i+1},\{\boldsymbol{r}\}^{\text {curr }}$ and $\{\boldsymbol{r}\}^{\text {rev }}$ are identical to within numerical accuracy, while if a plastic event occurs, the difference between the two configurations can be used to characterize the event. To do so, we follow the approach described in Ref. [27], which can be briefly summarized as follows. SW-like potentials contain only shortrange local energy terms. Hence a local energy measure can be defined to quantify the changes between an initial and a final configuration, in the present case, between $\{\boldsymbol{r}\}^{\text {curr }}$ and $\{\boldsymbol{r}\}^{\text {rev }}$. The energy variation around an atom $i_{a}$ is given by the squares of the potential energy contributions in which the atom is involved. For an SW potential, the sums run over the twoand three-body terms involving the neighbors of atom $i_{a}$ :

$$
\begin{aligned}
\operatorname{PE}_{\mathrm{at}}\left(i_{a}\right)= & \left(\sum_{j_{a}}\left|V_{i_{a}, j_{a}}^{2 b}\left(\{\boldsymbol{r}\}^{\mathrm{curr}}\right)-V_{i_{a}, j_{a}}^{2 b}\left(\{\boldsymbol{r}\}^{\mathrm{rev}}\right)\right|^{2}\right. \\
& \left.+\sum_{j_{a}} \sum_{k_{a}}\left|V_{j_{a}, i_{a}, k_{a}}^{3 b}\left(\{\boldsymbol{r}\}^{\mathrm{curr}}\right)-V_{j_{a}, i_{a}, k_{a}}^{3 b}\left(\{\boldsymbol{r}\}^{\mathrm{rev}}\right)\right|^{2}\right)^{1 / 2},
\end{aligned}
$$

where $V_{i_{a}, j_{a}}^{2 b}$ is the two-body energy due to the interaction between atom $i_{a}$ and atom $j_{a}$, and $V_{j_{a}, i_{a}, k_{a}}^{3 b}$ the three-body contribution from the triplet $j_{a}, i_{a}, k_{a}$ centered on $i_{a}$.

With the initial and final configurations being related by an incremental strain cycle, we associate $\operatorname{PE}_{\text {at }}\left(i_{a}\right)$ with an atomic plastic activity. Indeed, $\operatorname{PE}_{\mathrm{at}}\left(i_{a}\right)$ is 0 within numerical accuracy if no irreversible rearrangement occurs between $\gamma_{i}$ and $\gamma_{i+1}$, and conversely, $\operatorname{PE}_{\mathrm{at}}(i a)$ is positive and peaked at the rearrangement core if a plastic deformation occurs during the strain cycle.

From $\mathrm{PE}_{\mathrm{at}}\left(i_{a}\right)$, a continuous field $\mathrm{PE}(\boldsymbol{r})$ is obtained using the coarse-graining procedure outlined in Ref. [35] with

$$
\mathrm{PE}(\boldsymbol{r})=\sum_{i_{a}} \mathrm{PE}_{\mathrm{at}}\left(i_{a}\right)\left(\frac{1}{\left(\pi \omega^{2}\right)^{3 / 2}} \exp -\frac{\left|\boldsymbol{r}-\boldsymbol{r}_{i_{a}}\right|^{2}}{\omega^{2}}\right),
$$




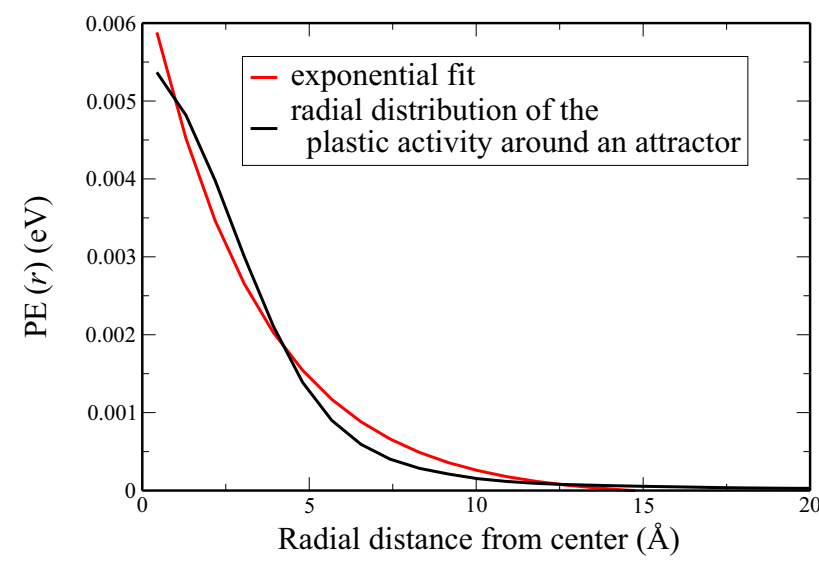

FIG. 2. Size of the main plastic event at $\gamma=0.168$ in an SW sample. Black curve: radial distribution of plastic energy $\mathrm{PE}(r)$ around the attractor. Red curve: exponential fit with a characteristic size $\lambda=3.36 \AA$.

where $\omega$ is the Gaussian width, chosen close to the average first-neighbor distance $(\omega=2.6 \AA)$. The field $\operatorname{PE}(\boldsymbol{r})$ is then subjected to a topological analysis reminiscent of the Bader charge analysis in quantum chemistry [36]. Practically, $\mathrm{PE}(\boldsymbol{r})$ is written on a $3 \mathrm{D}$ grid $(100 \times 100 \times 100)$ and its attractors are found along with their associated basins. The attractors are the local maxima of $\operatorname{PE}(\boldsymbol{r})$, while a basin is defined as a region of space traversed by all the gradient paths that terminate at a given attractor. Technical details concerning the determination of the maxima and basins are given in Ref. [27].

The relative importance of the attractors can be evaluated by integrating $\mathrm{PE}(\boldsymbol{r})$ in each basin. We later refer to this quantity as the plastic intensity of an attractor. From this analysis, we now identify the relevant plastic events with the attractors bearing a significant plastic intensity. Even in the absence of plastic events during a step increment, the initial and reversed configurations will not be strictly equivalent because of the finite numerical accuracy. This gives rise to thousands of very small and insignificant attractors that have to be removed from the analysis. To do so, we have applied two criteria discussed and tested in Ref. [27]. The first depends on the heterogeneities in the distribution of the attractor intensity and the second selects additional attractors whenever they are close to atoms that display a nonaffine displacement larger than $0.01 \AA$.

After this first selection the total number of events found in the range $0<\gamma<0.3$ is about 13000 for an SW sample. To obtain a computationally efficient method while keeping the most relevant plastic events, we then order at each strain increment the detected plastic events as a function of their plastic intensity and keep only the largest events, with a plastic intensity greater than $0.5 \mathrm{eV}$, until their sum represents more than $90 \%$ of the total plastic intensity. In this way, the selected plastic events carry more than $90 \%$ of the plastic intensity and their number is typically about 3500 for an SW sample and less $(\simeq 2500)$ for an SWM sample. With this technique the first detected plastic events always appear before $\gamma=0.002$.

A size can also be attributed to each plastic event by integrating $\mathrm{PE}(\boldsymbol{r})$ over the angular coordinates to obtain an average radial $\operatorname{PE}(r)$ function. As illustrated in Fig. 2,
$\mathrm{PE}(r)$ can be fitted using an exponential function, $f(r)=$ $a+b \exp \left(-\frac{r}{\lambda}\right)$. This fit does not need to faithfully represent the detailed structure of $\operatorname{PE}(r)$ since its sole purpose is to estimate the size of the plastic event. On average, the plastic event sizes found in the SW and SWM samples are $\lambda=4.7$ and $4.1 \AA$, respectively. However, whenever a shear band forms, $\mathrm{PE}(r)$ exhibits several large maxima that are described within the present analysis as a collection of separated plastic events. Within the shear band, larger plastic events are usually found, with typical sizes of the order of $\lambda \simeq 10-20 \AA$. More details on the variation of the plastic event size as a function of the strain can be found in Ref. [27]. These estimates of the plastic event sizes are used as input for the Eshelby fits in Sec. IV.

\section{EXTRACTING THE PLASTIC ENERGY}

The incremental strain cycles allow us to identify the plastic contribution to the mechanical energy of the systems. While a forward step may induce a plastic event, we assume that the reverse steps are always elastic. Therefore, if no plastic event occurs between $\gamma_{i}$ and $\gamma_{i+1}$, the energies of the current $\left(E_{i}^{\text {curr }}\right)$ and reversed $\left(E_{i}^{\mathrm{rev}}\right)$ configurations are the same to within numerical accuracy, while if a plastic event occurs, the energies are different and their difference corresponds to the plastic energy dissipated during the strain cycle. We may thus define an incremental plastic energy,

$$
\delta E_{i \rightarrow i+1}^{\mathrm{pl}}=E_{i}^{\mathrm{curr}}-E_{i}^{\mathrm{rev}},
$$

with the total plastic energy at strain $\gamma_{i}$ defined as $E_{i}^{\mathrm{pl}}=$ $\sum_{j=0}^{i-1} \delta E_{i \rightarrow i+1}^{\mathrm{pl}}$.

Similarly, the total mechanical work given to the system is

$$
W_{i}^{\text {mech }}=\int_{0}^{\gamma_{i}} \sigma_{x y}(\gamma) d \gamma \simeq \sum_{j=0}^{i-1} \sigma_{x y}\left(\gamma_{j}\right) \delta \gamma_{x y},
$$

where $\sigma_{x y}\left(\gamma_{j}\right)$ is the Irving-Kirkwood stress [37] in the current configuration calculated independently from the reverse step.

From the above definitions, one can reconstruct the potential energy of the system at the strain $\gamma_{i}$ as the amount of mechanical work brought by the imposed macroscopic deformation up to the strain $\gamma_{i}$ minus the energy $E_{i}^{\mathrm{pl}}$ dissipated through plasticity. These two components are plotted in Fig. 3 and their difference (dashed orange curve) reproduces the atomistic potential energy in output of the MD simulation (black curve) with a very good precision. This result validates our practical definition of the plastic energy increment through the difference between the current and the reverse energies in Eq. (3). Importantly, it also confirms the elastic nature of the reverse step.

Thanks to the latter property, the shear modulus can be estimated as $G\left(\gamma_{i}\right)=G_{i}=\left(\sigma_{x y}\left(\gamma_{i}\right)-\sigma_{x y}^{\mathrm{rev}}\left(\gamma_{i-1}\right)\right) / \delta \gamma_{x y}$. We checked that this technique is equivalent within $\simeq 5 \%$ to the more precise method where the shear modulus is calculated from small elastic strain increments $\left(\delta \gamma_{x y}=10^{-4}\right)$ around the configurations with a linear interpolation between stress and strain. Unless otherwise mentioned, the shear modulus is calculated using the small-strain-increment technique. Similarly, the bulk modulus and Poisson's ratio are calculated using the same small-increment technique. In the next section, we 


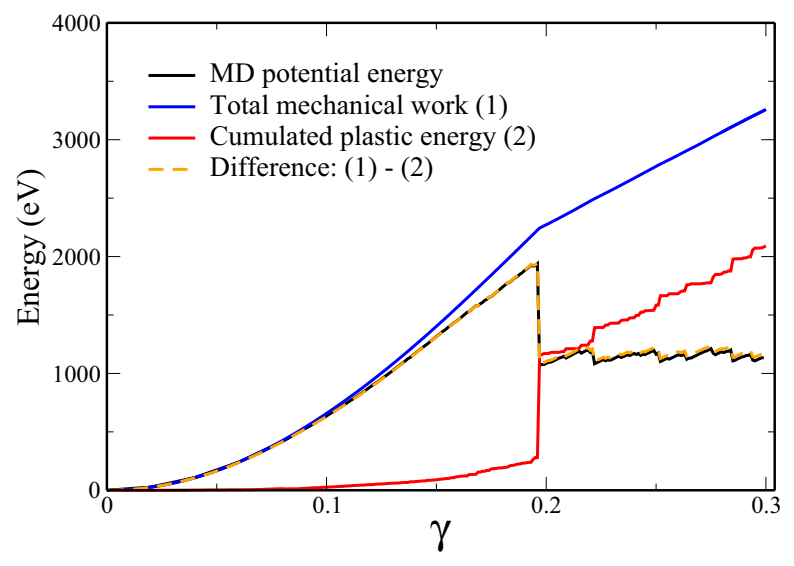

FIG. 3. Energy decomposition in an SW sample. Black curve: $E$, potential energy from the atomistic calculation. Red curve: $E^{\mathrm{pl}}$, plastic energy contribution. Blue curve: $W^{\text {mech }}$, mechanical work from Eq. (4). Dashed orange curve: difference $W^{\text {mech }}-E^{\mathrm{pl}}$, which compares accurately with $E$.

further develop the idea of localized plastic event analysis and extract the distribution of Eshelby inclusions from the atomistic simulations.

\section{FITTING ESHELBY INCLUSIONS FROM ATOMISTIC CONFIGURATIONS}

\section{A. Fitting displacements}

In order to keep the fitting procedure as practical as possible, we used the simplest displacement expressions, which correspond to the spherical Eshelby inclusion in an infinite isotropic elastic solid. Using such expressions implies several approximations in building the continuum representation of the MD results. First, the model uses homogeneous elastic constants, i.e., equal values inside and outside the inclusions, although amorphous solids are known to be elastically heterogeneous at mesoscopic length scales [26]. Second, the shape of the inclusions is assumed to be spherical, which may be an oversimplification. However, we show in the following that this procedure reproduces with fidelity the mechanical behavior of the amorphous solids under shear.

The fitting procedure relies on the displacement field due to a stress-free strain transformation defined by the tensor $\overline{\overline{\epsilon^{T}}}$ inside a spherical inclusion of radius $a$ embedded in an infinite body with a shear modulus $G$ and Poisson's ratio $v$. We first define the stress transformation tensor $\overline{\bar{p}}$ (implicit Einstein summation applies over repeated indices in Eqs. (5)-(9):

$$
p_{i j}=2 G\left\{\epsilon_{i j}^{T}+v \delta_{i j} \frac{\epsilon_{k k}^{T}}{(1-2 v)}\right\} .
$$

The displacement and stress fields reproduced from Ref. [38] outside of the inclusion are

$$
\begin{aligned}
u_{i}= & \frac{a^{3}}{4(1-v) G}\left\{\frac{\left(2 p_{i k} x_{k}+p_{k k} x_{i}\right)}{15 R^{5}}\left(3 a^{2}-5 R^{2}\right)\right. \\
& \left.+\frac{p_{j k} x_{j} x_{k} x_{i}}{R^{7}}\left(R^{2}-a^{2}\right)+\frac{4(1-v) p_{i k} x_{k}}{3 R^{3}}\right\},
\end{aligned}
$$

$$
\begin{aligned}
\sigma_{i j}^{\text {out }}= & \frac{a^{3}}{2(1-v) R^{3}}\left\{\frac{p_{i j}}{15}\left(10(1-2 v)+6 \frac{a^{2}}{R^{2}}\right)\right. \\
& +\frac{p_{i k} x_{k} x_{j}+p_{j k} x_{k} x_{i}}{R^{2}}\left(2 v-2 \frac{a^{2}}{R^{2}}\right) \\
& +\frac{\delta_{i j} p_{k k}}{15}\left(3 \frac{a^{2}}{R^{2}}-5(1-2 v)\right) \\
& +\frac{\delta_{i j} p_{k l} x_{k} x_{l}}{R^{2}}\left((1-2 \nu)-\frac{a^{2}}{R^{2}}\right)-\frac{x_{i} x_{j} p_{k l} x_{k} x_{l}}{R^{4}} \\
& \left.\times\left(5-7 \frac{a^{2}}{R^{2}}\right)+\frac{x_{i} x_{j} p_{k k}}{R^{2}}\left(1-\frac{a^{2}}{R^{2}}\right)\right\} .
\end{aligned}
$$

Inside the inclusion, the solution for the strain is homogeneous and accounts for the relaxation of the transformation strain $\epsilon_{k l}^{T}$ in the presence of the surrounding elastic matrix,

$$
\epsilon_{i j}^{\mathrm{in}}=S_{i j k l} \epsilon_{k l}^{T},
$$

where $\overline{\bar{S}}$ is the Eshelby tensor for the spherical inclusion, which depends only on Poisson's ratio. The stress field inside the inclusion is also homogeneous and accounts for the fact that the local stress-free reference has changed to the transformation strain:

$$
\sigma_{i j}^{\text {in }}=C_{i j k l} \epsilon_{k l}^{\text {in }}-p_{i j}=C_{i j k l}\left(\epsilon_{k l}^{\mathrm{in}}-\epsilon_{k l}^{T}\right) .
$$

Inside an inclusion, the atomistic displacements due to the plastic rearrangements involve atomic bond disruption and/or formation and depend strongly on the local atomic structure. The atomic displacements inside the inclusions are consequently excluded from the fit. Only the displacements outside the inclusions are adjusted using Eq. (6) as a fitting function.

For the fit, at a given strain $\gamma_{i}$, we use the energy analysis presented in Sec. II B to identify the location and size of the relevant plastic events, counted by $e$ from 1 to $N_{\text {sel }}$, the total number of selected events at this strain. We also identify the center of these events, identified by $i_{c}(e)$, the index of the atom closest to the center of the plastic event $e$. The atomic displacements $d_{i, i_{a}}$ of atoms $i_{a}$ in direction $i$ are then computed between the current and the reversed configurations, $d_{i, i_{a}}=$ $r_{i, i_{a}}^{\mathrm{rev}}-r_{i, i_{a}}^{\mathrm{curr}}$, and an objective function $f$ is defined as

$$
f=\sum_{i_{a}=1}^{N^{\text {out }}} \sum_{i} \frac{1}{2}\left|d_{i, i_{a}}-\sum_{e=1}^{N_{\text {sel }}} u_{i}\left(\boldsymbol{r}_{i_{a}}^{\text {curr }}-\boldsymbol{r}_{i_{c}(e)}^{\text {curr }}, \overline{\overline{\epsilon^{T}}}(e)\right)\right|^{2},
$$

where $i_{a}$ runs over the $N^{\text {out }}$ atoms outside the inclusions $\left(\left|\boldsymbol{r}_{i_{a}}^{\text {curr }}-\boldsymbol{r}_{i_{c}(e)}^{\text {curr }}\right|>a(e)\right)$. The size of event $e, a(e)$, from Eqs. (6) and (7), is not fitted but taken as the characteristic size $\lambda(e)$ presented in Sec. II B. The shear modulus and Poisson's ratio are determined for each strain from the small-strain technique mentioned above. The objective function, $f$, is then minimized with respect to $\left\{\overline{\overline{\epsilon^{T}}}(e), e=1, N_{\text {sel }}\right\}$ using damped MD. The symmetry $\epsilon_{i j}^{T}=\epsilon_{i i}^{T}$ is imposed in the fit, leading to six variable parameters per selected plastic inclusion. Also, to account for the periodic boundary conditions, we used the minimum image convention for the displacements $u_{i}$. A more detailed analysis of the fit convergence as a function of the number of periodic images used for $u_{i}$ is presented in the Appendix. 

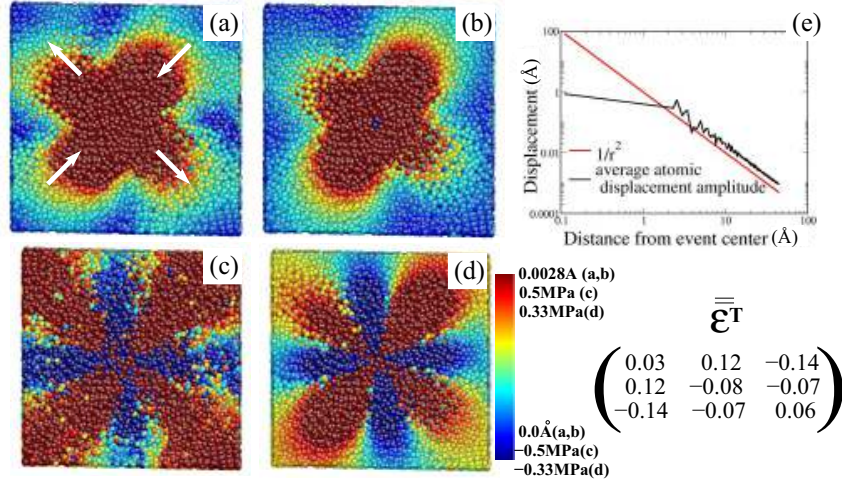

FIG. 4. Plastic event extracted at $\gamma=0.02$ in an SWM sample. (a) Amplitude of the atomic displacement field in an $(x, y)$ plane containing the center of the event (white arrows show the main directions of displacement). (b) Amplitude of the continuum displacement field fitted from Eq. (6) using $a(e)=\lambda(e)=2.65 \AA$. (c) Atomic shear stress field $\sigma_{x y}$. (d) Continuum shear stress from Eq. (7). Atoms with values out of the color maps are shown with saturated colors. (e) Black line: angular average of the atomistic displacement amplitude as a function of the distance from the event center. Red line: $1 / r^{2}$ function, for comparison. The transformation strain tensor associated with the plastic event is shown at the lower right.

We give an illustrative example of the atomic displacements due to a selected plastic event together with the result of the fit in Fig. 4. To isolate a single event, we first identified a highplastic-intensity event, found at $\gamma=2 \%$ in an SWM sample. To make sure that the displacement field was due only to this event, we started from the atomic coordinates in the current configuration and added the displacements $d_{i, i_{a}}=r_{i, i_{a}}^{\text {rev }}-r_{i, i_{a}}^{\text {curr }}$ only to the atoms inside the spherical inclusion. These atoms were then held fixed, while all others were relaxed with damped MD. As shown in Fig. 4(a), this artificial procedure triggered only the selected event. The cross-shaped atomistic displacement field is typical of the quadrupolar symmetry of a local shear transformation. The atomistic displacement amplitude [Fig. 4(e)] shows a $\frac{1}{r^{2}}$ radial dependence, which matches the Eshelby displacement field in Eq. (6). The structural features are also well reproduced by the continuum fit shown in Fig. 4(b). Comparison between Figs. 4(c) and 4(d) also evidences a good agreement between the atomistic and the continuum stress fields. Generally, larger local fluctuations appear in the atomistic plots, while the continuum fit slightly underestimates the amplitude of the atomic stress field. The optimized transformation strain tensor $\overline{\overline{\epsilon^{T}}}$ is given at the lower right in Fig. 4 and shows the dominant shear character of this event. At the atomic scale, amorphous samples are not homogeneous or isotropic, which explains that the symmetry of the shear components in this event may differ from the pure shear $(x y)$ symmetry of the imposed deformation.

\section{B. Quality of the fit and relevant physical parameters}

\section{Transformation strain tensors}

The outcome of the fits are the six independent components of the transformation strain tensor $\overline{\overline{\epsilon^{T}}}(e)$ for all the selected
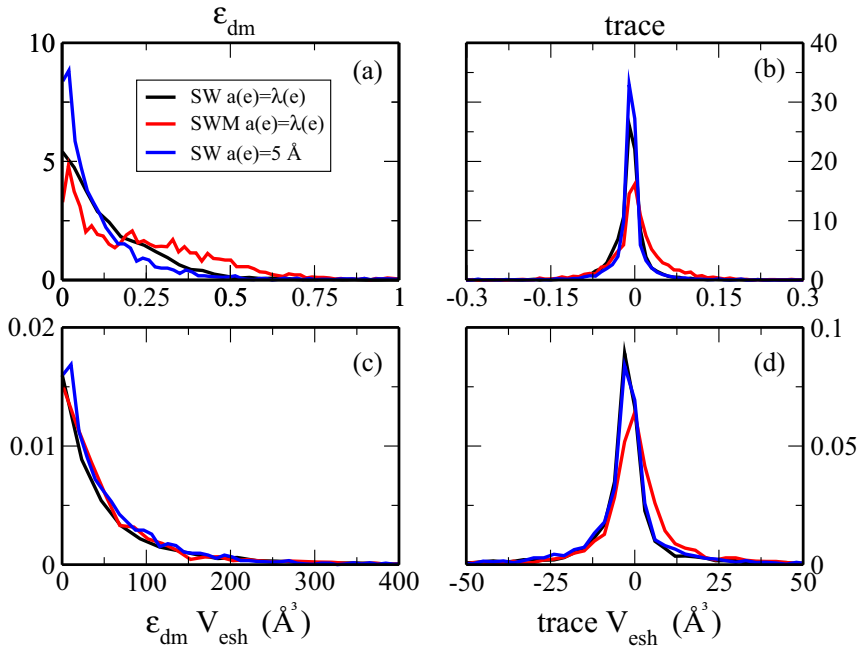

FIG. 5. Distributions of (a) $\epsilon_{\mathrm{dm}}$ and (b) trace components of the fitted transformation strain tensors. Black curve: SW sample with a variable inclusion radius $[a(e)=\lambda(e)]$. Red curve: same in an SWM sample. Blue curve: SW sample with a fixed inclusion radius $[a(e)=5 \AA]$. (c, d) Distributions of $\epsilon_{\mathrm{dm}} V_{\text {esh }}$ and $\operatorname{Tr}\left[\overline{\overline{\epsilon^{\mathrm{T}}}}\right] \mathrm{V}_{\text {esh }}$.

events in the range $0<\gamma<0.3$. To synthesize these results, we rewrite each tensor as the sum of a hydrostatic tensor $\epsilon_{i, j}^{T, h}(e)=\delta_{i j} \frac{\sum_{k} \epsilon_{k k}^{T}(e)}{3}$ plus a deviatoric part, $\overline{\overline{\epsilon^{T, d}}}(e)=\overline{\overline{\epsilon^{T}}}(e)-$ $\overline{\overline{\epsilon^{T, h}}}(e)$. In Fig. 5(a), we show the distribution of $\epsilon_{\mathrm{dm}}(e)$, the maximum shear component of the strain tensor, equal to the largest difference between the eigenvalues of $\overline{\overline{\epsilon^{T, d}}}(e)$. In Fig. 5(b) we also give the distribution of the hydrostatic traces. These raw transformation strain tensors depend on the size of each event $\lambda(e)$, which is estimated after an exponential fit of the local plastic activity outlined in Sec. II B. Indeed the results shown in Figs. 5(a) and 5(b) for the same SW sample but for different input sizes lead to different distributions of the transformation strain components. This point prompts some caution in the interpretation of the fit output, but as suggested from Eq. (6), the bare strain transformation tensor only partially defines the displacement field due to an inclusion. A physically more relevant local quantity should be built from the product of the strain tensor with the inclusion volume $V_{\text {esh }}$, which also appears in the stress drops associated with the plastic events [see Eq. (7) and Sec. V A]. When the factor $V_{\text {esh }}(e)$ is taken into account, we can check in Figs. 5(c) and 5(d) that the distributions using different size parameters collapse into a single curve. This indicates that the relevant quantity to characterize an Eshelby inclusion is the product $\overline{\overline{\epsilon^{T}}} V_{\text {esh }}$ rather than the bare transformation strain tensor. At this point, several observations can be extracted from these distributions. First, the average amplitude of the traces times the Eshelby inclusion volumes in Fig. 5 (d) are all of the order of $\simeq 2 \AA^{3}$, but they are positive for the SWM sample and negative for the SW sample. This suggests an average compression in the Eshelby's center upon plastic rearrangement in the SW samples, while an average dilatation occurs in the SWM samples. The $\epsilon_{\mathrm{dm}} V_{\text {esh }}$ distributions in Fig. 5(c) can approximately be represented by an exponential function with an average value of about 


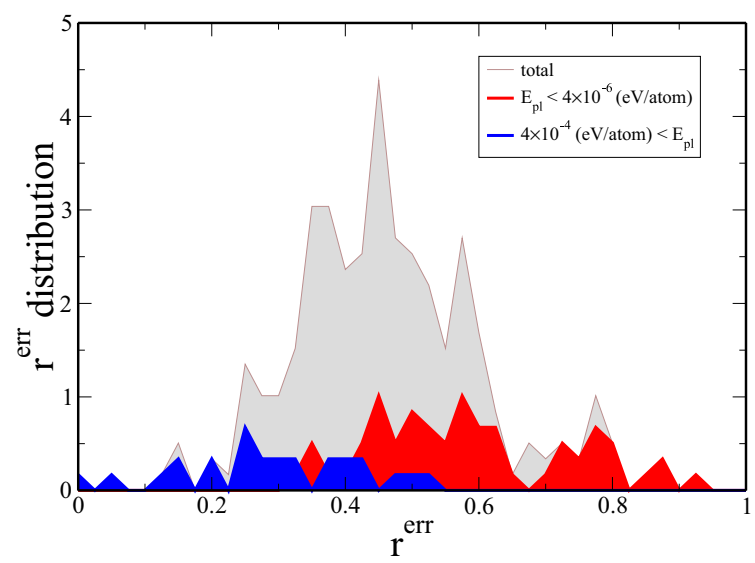

FIG. 6. Error histograms from the fitting procedure in an SW sample. Red area: $r^{\text {err }}$ histogram for events with a low plastic energy (high $r^{\text {err }}$ ). Blue area: $r^{\text {err }}$ histogram for events with a high plastic energy (low $\left.r^{\text {err }}\right)$. Gray area: $r^{\text {err }}$ total histogram.

$45 \AA^{3}$, larger than the average hydrostatic components, consistent with the predominantly shear nature of the plastic events. The similitude of the $\epsilon_{\mathrm{dm}} V_{\mathrm{esh}}$ distributions between SW and SWM samples and the larger values of $\epsilon_{\mathrm{dm}}$ in Fig. 5(a) imply that, on average, the plastic rearrangements in SWM samples have smaller plastic cores with larger shear components. An analysis of the eigenvectors of $\overline{\overline{\epsilon^{T}}}(e)$ reveals that, after a few hundred plastic events $(\gamma \leqslant 0.1)$, their average eigendirections match the eigendirections expected from pure shear events in the $(x y)$ plane. However, as illustrated in Fig. 4, large fluctuations may locally occur in specific events.

\section{Precision of the fit}

Several sources may contribute to the error in the fit, mainly the local fluctuations due to the discrete atomic structure, the choice of a restricted list of plastic events with spherical shapes, the use of the minimum image convention, and the assumption of homogeneous elastic constants. Also, the boundary conditions may contribute since the latter are expected to result additional fields, which vary slowly compared to the infinite medium solution in Eq. (6) and cannot be represented by Eshelby solutions.

To estimate the precision of the fit, we define the $r^{\text {err }}$ ratio, which measures the relative error by dividing the value of the objective function after the fitting procedure, $f_{\min }$, by $f_{0}=$ $\sum_{i_{a}=1}^{N^{\text {out }}} \sum_{i} \frac{1}{2}\left|d_{i, i_{a}}\right|^{2}$, the value of the objective function in the absence of inclusions:

$$
r^{\mathrm{err}}=\frac{f_{\mathrm{min}}}{f_{0}}
$$

The best fit quality is obtained for $r^{\mathrm{err}}=0$ when the displacement field is fully represented by Eshelby inclusions, while, on the other hand, $r^{\text {err }}=1$ indicates that the selected Eshelby inclusions do not participate in the displacement field. Therefore, the $r^{\text {err }}$ ratio also provides a measure of the contribution of the Eshelby inclusions to the total displacement field. In Fig. 6 the main curve defining the gray region shows a histogram of the fit error $r^{\text {err }}$ calculated in the range

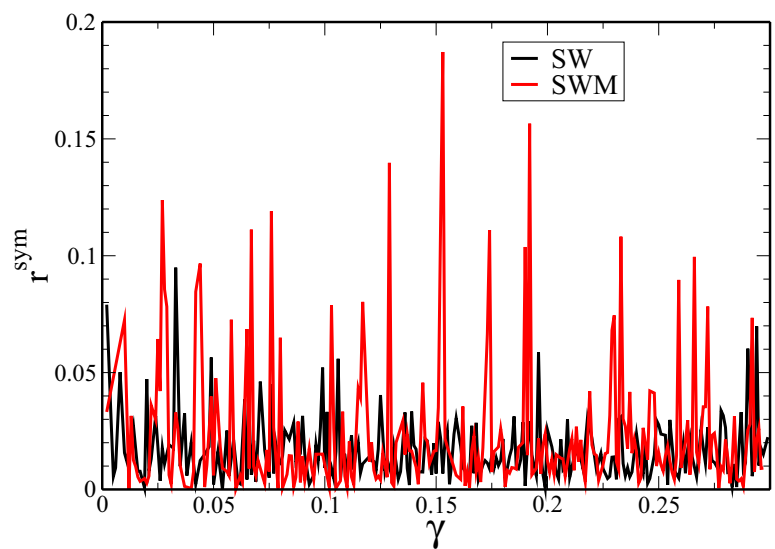

FIG. 7. $r^{\text {sym }}$ ratio from Eq. (12) as a function of shear strain for an SW sample (black curve) and an SWM sample (red curve).

$0<\gamma<0.3$ for an SW sample. The average value is around 0.47 but the error distribution is widely spread. Splitting the histogram into three pieces, corresponding to configurations with low, average, and high plastic energies, shows that the fit gives a better representation when the plastic energy is high, the reason being that, in this case, atomic displacements are dominated by the Eshelby inclusions. On the contrary, when only a few and/or weak plastic events occur, the main displacements originate from the local fluctuations due to the discrete atomic structure, which cannot be represented by the continuum expressions. The same observations hold for SWM samples, with, however, a smaller average $r^{\text {err }}$ value, around 0.4 .

We conclude therefore that the significant plastic events can be satisfactorily represented by a continuum fit based on Eshelby inclusions, while the long-wavelength displacement fields due to the boundary conditions and the fluctuations due to the local atomic environments are filtered out.

\section{Symmetry of plastic events}

To gauge the relative importance of the shear and hydrostatic components in the displacement field due to the Eshelby inclusions we construct the ratio $r^{\mathrm{sym}} \cdot r^{\mathrm{sym}}$ is calculated for each strain $\gamma_{i}$ as the sum of the displacements squared due to the hydrostatic components $\overline{\overline{\epsilon^{T, h}}}$ divided by the sum of the displacements squared originating from the deviatoric part $\overline{\overline{\epsilon^{T, d}}}$ :

$$
r^{\mathrm{sym}}=\frac{\sum_{i_{a}=1}^{N_{\text {out }}} \sum_{i}\left|\sum_{e=1}^{N_{\text {sel }}} u_{i}\left(\boldsymbol{r}_{i_{a}}^{\mathrm{ini}}-\boldsymbol{r}_{i_{c}(e)}^{\mathrm{ini}}, \overline{\overline{\epsilon^{T, h}}}(e)\right)\right|^{2}}{\sum_{i_{a}=1}^{N_{\text {out }}} \sum_{i}\left|\sum_{e=1}^{N_{\text {sel }}} u_{i}\left(\boldsymbol{r}_{i_{a}}^{\mathrm{ini}}-\boldsymbol{r}_{i_{c}(e)}^{\text {ini }}, \overline{\overline{\epsilon^{T, d}}}(e)\right)\right|^{2}} .
$$

The symmetry ratio $r^{\text {sym }}$ is shown in Fig. 7 for both an SW and an SWM sample. The amplitude of $r^{\text {sym }}$ is always lower than 0.2 , indicating that plasticity is dominated by events bearing a strong shear character. Interestingly, we note that larger $r^{\text {sym }}$ values are found in the SWM sample, meaning that the relative hydrostatic contributions in the displacements are stronger with this potential. This can be qualitatively understood assuming that the energy cost for local volume and shear deformations follows the same trends as the bulk and shear moduli, respectively. At $\gamma=0$, the SW and SWM 
samples have roughly the same bulk modulus $(B \simeq 100 \mathrm{GPa})$ but their shear moduli differ by a factor of close to $2\left(G_{\mathrm{SW}} \simeq\right.$ $35 \mathrm{GPa}$ and $G_{\mathrm{SWM}} \simeq 60 \mathrm{GPa}$ ). The energy difference between shear and volume deformations is therefore lower in the SWM samples, leading to a larger proportion of volume variation upon plastic rearrangements, in agreement with Fig. 7. At this point, we have shown that the relevant features of plastic displacements in sheared bulk amorphous models can be consistently represented by a simple Eshelby inclusion model where the plastic events show a dominant shear character. However, the contribution of hydrostatic displacements may depend on the loading condition, for instance, under pressure confinements of particular interest for silica [39-41]. In the next section, we examine to what extent the superposition of these inclusions can reproduce the stress-strain curves predicted by the atomistic calculations.

\section{STRAIN-STRESS CURVES FROM ESHELBY INCLUSIONS}

\section{A. Shear stress}

Starting from a general elastoplastic approach, the measurable strain variation $\delta_{i} \epsilon_{x y}$ between two successive steps $\gamma_{i}$ and $\gamma_{i+1}$ is decomposed into an elastic and a plastic part:

$$
\delta_{i} \epsilon_{x y}=\delta_{i} \epsilon_{x y}^{\mathrm{el}}+\delta_{i} \epsilon_{x y}^{\mathrm{pl}} .
$$

The associated shear stress variation is

$$
\delta_{i} \sigma_{x y}=2 G\left(\gamma_{i}\right) \delta_{i} \epsilon_{x y}^{\mathrm{el}},
$$

which leads to the stress decomposition

$$
\delta_{i} \sigma_{x y}=2 G\left(\gamma_{i}\right) \delta_{i} \epsilon_{x y}-2 G\left(\gamma_{i}\right) \delta_{i} \epsilon_{x y}^{\mathrm{pl}}=\delta_{i} \sigma_{x y}^{*}-\delta_{i} \sigma_{x y}^{\mathrm{pl}} .
$$

In our calculations, $\delta_{i} \epsilon_{x y}=\delta \gamma_{x y} / 2$ and $\delta_{i} \sigma_{x y}$ are directly calculated from the atomistic simulations as the difference in strain and shear stress between the current configurations at $\gamma_{i}$ and $\gamma_{i+1} . \delta_{i} \sigma_{x y}^{*}$ is the stress variation that would be obtained in the absence of plasticity. In the following, we call it the elastically extrapolated shear stress. $\delta_{i} \sigma_{x y}^{*}$ can be estimated in two equivalent ways, by using either the elastic constant $\delta_{i} \sigma_{x y}^{*}=G\left(\gamma_{i}\right) \delta \gamma_{x y}$, or the stress variation during the reverse

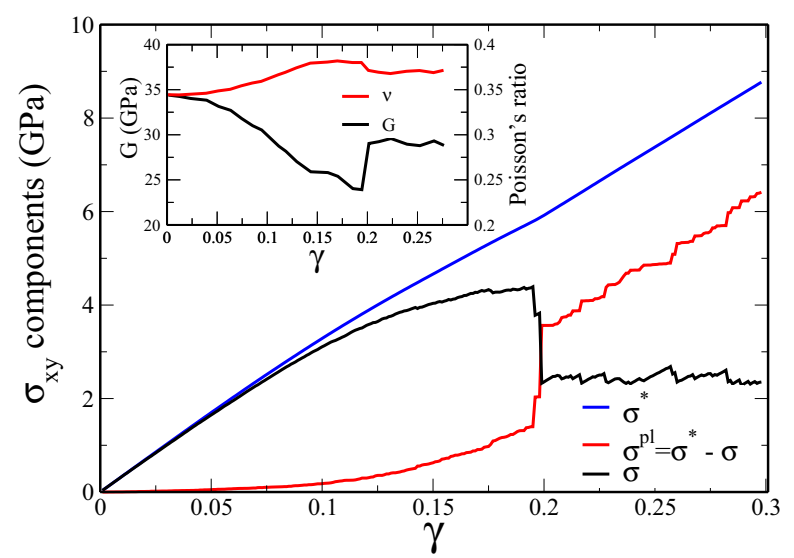

FIG. 8. Shear stress decomposition in an SW sample. Black curve: atomistic calculations. $\sigma_{x y}$. Red curve: $\sigma^{\mathrm{pl}}=\sigma_{x y}^{*}-\sigma_{x y}$. Blue curve: elastically extrapolated shear stress $\sigma_{x y}^{*}$. Inset: Shear modulus $G$ and Poisson's ratio $v$ as a function of the imposed strain. step $\delta_{i} \sigma_{x y}^{*}=\sigma_{x y}\left(\gamma_{i}\right)-\sigma_{x y}^{\text {rev }}\left(\gamma_{i-1}\right)$. These two methods lead to numerically indistinguishable stresses, $\sigma_{x y}^{*}\left(\gamma_{i}\right)=\sum_{j=0}^{i-1} \delta_{j} \sigma_{x y}^{*}$, shown in Fig. 8 (blue curve) for an SW sample, together with the total shear stress $\sigma_{x y}\left(\gamma_{i}\right)=\sum_{j=0}^{i-1} \delta_{j} \sigma_{x y}$ directly computed from the atomistic simulation. The plastic stress is then deduced from the difference $\sigma_{x y}^{\mathrm{pl}}\left(\gamma_{i}\right)=\sigma_{x y}^{*}\left(\gamma_{i}\right)-\sigma_{x y}\left(\gamma_{i}\right)$, which can be compared to the plastic shear stress due to the Eshelby inclusions. Taking into account the sign in Eq. (15), the contribution of a single inclusion $-\delta \sigma_{x y}^{\mathrm{pl}, \mathrm{esh}}(e)$ is given by the average of the stress field obtained outside [Eq. (7)] and inside [Eq. (9)] the Eshelby inclusion. The angular sum over the terms of $\sigma_{i j}^{\text {out }}$ in Eq. (7) vanishes, while $\sigma_{i j}^{\text {in }}$ is constant inside the inclusion sphere, leading to

$$
\begin{aligned}
\delta \sigma_{x y}^{\mathrm{pl}, \mathrm{esh}}(e) & =-\frac{V_{\mathrm{esh}}(e)}{V_{\text {cell }}} \sigma_{x y}^{\mathrm{in}}(e) \\
& =\frac{2 G\left(\gamma_{i}\right) V_{\mathrm{esh}}(e)}{V_{\text {cell }}}\left(\epsilon_{x y}^{T}(e)-\epsilon_{x y}^{\mathrm{in}}(e)\right),
\end{aligned}
$$

where $V_{\mathrm{esh}}(e)$ is the volume of the spherical inclusion $e$ and $V_{\text {cell }}$ the volume of the simulation cell.

The expression in Eq. (16), however, assumes an Eshelby inclusion in an infinite medium, while the simulations are performed at a fixed and finite volume with periodic boundary conditions. During a plastic event, the total shear strain increment calculated from the Eshelby solution over the cell is $\epsilon_{x y}^{\text {esh,tot }}=\sum_{e=1}^{N_{\text {sel }}} \frac{V_{\text {esh }}(e)}{V_{\text {cell }}} \epsilon_{x y}^{\text {in }}(e)$. However, the fixed cell calculation imposes a zero total strain. This requires a homogeneous correction, $-\epsilon_{x y}^{\text {esh,tot }}$, to recover a strain variation compatible with the boundary conditions. The shear stress drop from a single inclusion accounting for the boundary conditions is thus

$$
\begin{aligned}
\delta \sigma_{x y}^{\mathrm{pl}, \mathrm{esh}+\mathrm{bc}}(e) & =\delta \sigma_{x y}^{\mathrm{pl}, \mathrm{esh}}(e)+2 G\left(\gamma_{i}\right) \epsilon_{x y}^{\mathrm{esh}, \mathrm{tot}} \\
& =\frac{2 G\left(\gamma_{i}\right) V_{\mathrm{esh}}(e)}{V_{\text {cell }}}\left(\epsilon_{x y}^{T}(e)-\epsilon_{x y}^{\mathrm{in}}(e)+\epsilon_{x y}^{\mathrm{in}}(e)\right) \\
& =\frac{2 G\left(\gamma_{i}\right) V_{\mathrm{esh}}(e)}{V_{\text {cell }}} \epsilon_{x y}^{T}(e) .
\end{aligned}
$$

This correction cannot be neglected because the ratio between the corrected and the infinite-body expressions is high. One can easily show that this ratio equals $\left(1-\sum_{k l} S_{x y k l}\right)^{-1}=$ $\left(\frac{7-5 v}{15(1-v)}\right)^{-1}$, which is 1.86 with $v=0.34$ at $\gamma=0$ for the SW sample.

To test the quantitative accuracy of the plastic contribution estimated from the present continuum model, we write a model shear stress from the Eshelby inclusion fit as the difference between the elastically extrapolated stress and the plastic contribution from the Eshelby inclusions, taking into account the fixed cell condition:

$$
\sigma_{x y}^{\bmod }\left(\gamma_{i}\right)=\sum_{j=0}^{i-1} \delta_{j} \sigma_{x y}^{*}-\sum_{j=0}^{i-1} \delta_{j} \sigma_{x y}^{\mathrm{pl}, \mathrm{es}+\mathrm{bc}} .
$$

In the following we present the results of the model as described above and comment on the influence of the main parameters: the elastic constants, the inclusion size, and the structure of the strain transformation tensor $\overline{\overline{\epsilon^{T}}}$.

In Fig. 10, we show the results for an SW sample. The red curve was obtained with the original model, i.e., 


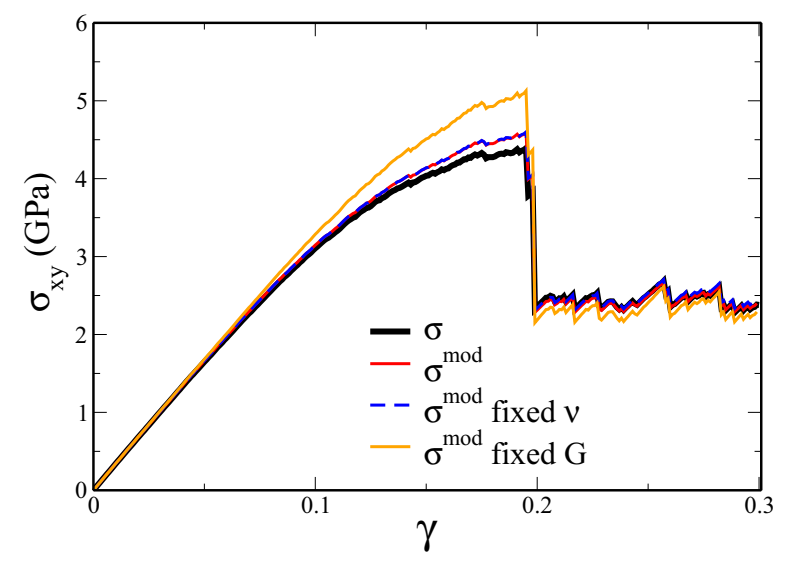

FIG. 9. Shear stress in an SW sample from the Eshelby inclusion model compared to atomistic results. Black curve: atomistic shear stress. Red curve: regular model, i.e., strain-dependent elastic constants and variable inclusion sizes $[a(e)=\lambda(e)]$. Dashed blue curve: regular model with fixed $v(\gamma=0)$. Orange curve: regular model with fixed $G(\gamma=0)$.

strain-dependent elastic constants and variable inclusion radii estimated from exponential fits of the radial plastic activities. This case has been considered in Fig. 8. The overall accuracy of $\sigma_{x y}^{\bmod }$ is very good, with a maximum error of about $4 \%$ at $\gamma=0.198$ just before the large stress drop associated with the formation of a persistent shear band. The dashed blue curve in Fig. 9 was obtained fixing Poisson's ratio to its value at $\gamma=0$ while keeping a varying shear modulus. The calculated shear stress is almost indistinguishable from the original model with variable $v$. The last curve (orange curve in Fig. 9) was obtained fixing the shear modulus at its value at $\gamma=0$ in both the elastic and the plastic contributions to $\sigma_{x y}^{\text {mod }}$. The discrepancy with respect to the atomistic reference is larger in this case, with a maximum error of $\simeq 18 \%$, showing that the nontrivial dependence of the shear modulus on the deformation is an important ingredient for a quantitative description.

Figure 10 displays shear stress plots for an SWM sample. The result for the original model (red curve) reproduces very satisfactorily the MD results, with a maximum error of the same order as for the SW potential $(+6 \%$ at $\gamma=0.298)$. The blue curve shows that a similar accuracy is reached when the size of the inclusions is fixed. As suggested by the strain distributions presented in Sec. III, this result confirms the relevant role of the quantity $\epsilon_{x y}^{T} V_{\text {esh. }}$. The shear stress curve obtained setting the diagonal elements of the transformation strain matrix to 0 is represented by the dashed orange curve in Fig. 10. A good agreement is again found, which confirms the central role of quadrupolar shear events in the plasticity of amorphous systems. These results validate the procedure used in the present inclusion model and confirm that modifications due to the shape of the inclusions and elastic heterogeneities are higher-order corrections that can be neglected. A deeper analysis of our results also provides some insight into the precision of the model. First, the model shear stress slightly overestimates the MD stress at low strain below the yield point. This probably comes from removing the smallest events from the selection list, which induces an underestimation of the plastic contribution. However, this effect is partially

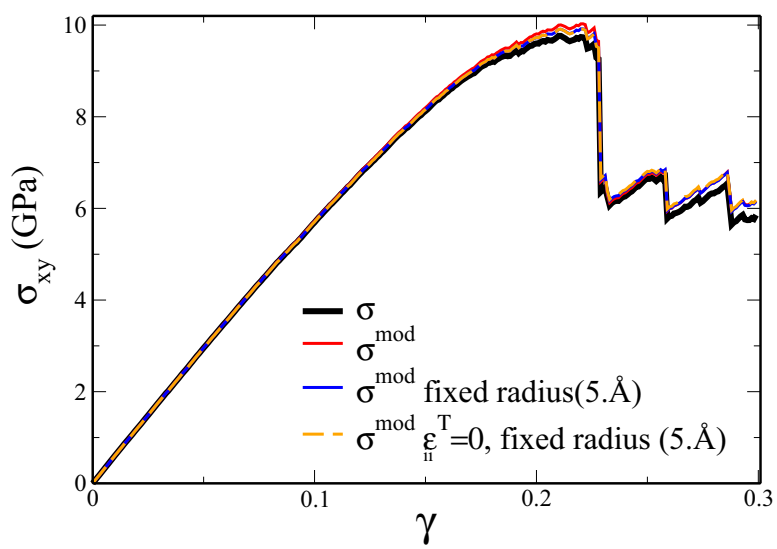

FIG. 10. Shear stress in an SWM sample from the Eshelby inclusion model compared to atomistic results. Black curve: atomistic shear stress. Red curve: regular model, i.e., strain-dependent elastic constants and variable inclusion sizes $[a(e)=\lambda(e)]$. Blue curve: strain-dependent elastic constants but a fixed inclusion radius $\left[a(e)=5 \AA\right.$. Dashed orange curve: $\overline{\overline{\epsilon^{T}}}$ diagonal elements set to 0 , strain-dependent elastic constants, and a fixed inclusion radius $[a(e)=5 \AA]$.

compensated by the overestimation of the stress drops due to the minimum image convention (see the Appendix), leading to the quantitative prediction of the MD shear stress in Figs. 9 and 10. In larger systems as shown in Fig. 11 ( $N=262144$ atoms, linear cell dimension $\approx 17 \mathrm{~nm})$, the stress drop overestimation due to the minimum image convention is reduced. The error is then dominated by the removal of the smallest events, leading to an overestimation of the stress. Since only $90 \%$ of the plastic activity is accounted for in the model, a correction can be proposed by including a scaling factor $\alpha$ to all shear stress drops $\delta_{j} \sigma_{x y}^{\mathrm{pl}, \mathrm{es}+\mathrm{bc}}$ in Eq. (18), with $1 \leqslant \alpha \leqslant 1$.1. Using this correction, an excellent quantitative agreement with the MD shear stress is achieved, as shown in Fig. 11, with $\alpha=1.05$ and 1.06 for the SW and SWM samples, respectively.

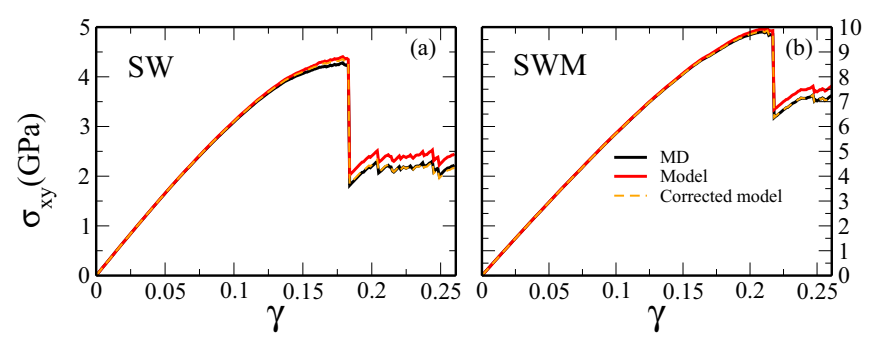

FIG. 11. Shear stress in large samples (262 144 atoms) from the Eshelby inclusion model compared to atomistic results: (a) SW sample; (b) SWM sample. Black curve: atomistic shear stress. Red curve: model with strain-dependent elastic constants and a fixed inclusion radius $[a(e)=5 \AA]$. Dashed orange curve: same as the red curve, with a scaling factor $\alpha$ applied to all plastic shear stress drops in Eq. (18). $\alpha=1.05$ for the SW sample in (a) and $\alpha=1.06$ for the SWM sample in (b). 


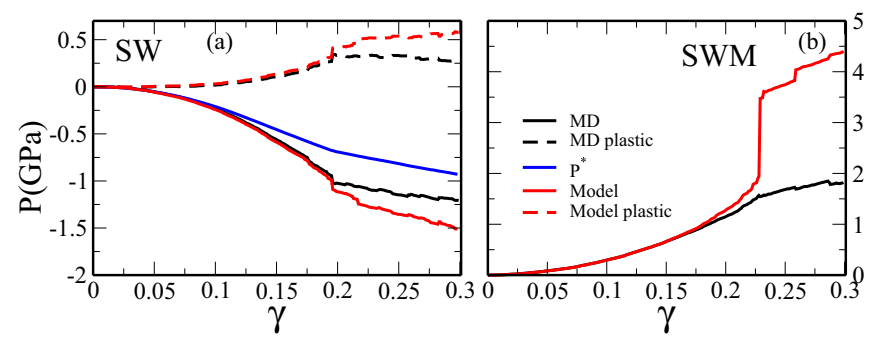

FIG. 12. Pressure variations in (a) an SW and (b) an SWM sample. Black curves: total (solid line) and plastic (dashed line) pressures from atomistic calculations. Red curves: same, for the model. Blue curve: elastically extrapolated $P^{*}$ contribution to the pressure from the atomistic calculations (SW sample).

\section{B. Pressure effects through the fit procedure}

Following the same technique as for the shear stress, we consider now the pressure variations due to plastic rearrangements. This task is more delicate than for shear stresses because, due to the present simple shear loading, no pressure variation is expected from linear elasticity. The latter is therefore a purely atomistic effect, which, however, displays a quadratic character as shown in Fig. 12. This variation is therefore probably due to nonzero third-order elastic constants.

We estimate the increment of the elastically extrapolated pressure by the difference $\delta P^{*}\left(\gamma_{i}\right)=P\left(\gamma_{i}\right)-P^{\text {rev }}\left(\gamma_{i-1}\right)$, where $P\left(\gamma_{i}\right)$ is the atomistic pressure in the current configuration at strain $\gamma_{i}$ and $P^{\text {rev }}\left(\gamma_{i-1}\right)$ is the pressure calculated after the reverse step at strain $\gamma_{i-1}$. The total elastically extrapolated pressure at strain $\gamma_{i}$ is then $P^{*}\left(\gamma_{i}\right)=\sum_{j=1}^{i-1} \delta P^{*}\left(\gamma_{j}\right)$. The plastic contribution is obtained as before as $P^{\mathrm{pl}}\left(\gamma_{i}\right)=$ $P^{*}\left(\gamma_{i}\right)-P\left(\gamma_{i}\right)$. The pressure given by the Eshelby inclusion model is calculated using Eq. (17) rewritten for the pressure.

The pressure in the SW sample in Fig. 12(a) follows the atomistic curve qualitatively well. In the SWM case in Fig. 12(b), the agreement remains satisfactory but large discrepancies are observed in a few configurations, which all coincide with the formation of a shear band. A detailed analysis shows that these large discrepancies arise because in a shear band the events are localized in a narrow region where a large number of atomic displacements are excluded from the fit since most atoms in this region are found inside the inclusion spheres. In this situation, large $\epsilon_{y y}$ and $\epsilon_{z z}$ can develop without inducing significant displacements in the fitted region outside the band. However, it is still possible to obtain a distribution of Eshelby inclusions that matches simultaneously the shear stress and the pressure by adding in the objective function of the fit an extra cost related to the plastic variation of the pressure $\delta P^{\mathrm{pl}}=P^{\mathrm{pl}}\left(\gamma_{i+1}\right)-P^{\mathrm{pl}}\left(\gamma_{i}\right)$,

$$
f^{P}=f+\frac{w_{P}}{G^{2}\left(\gamma_{i}\right)}\left[\delta P^{\mathrm{pl}}-\sum_{e=1}^{N_{\text {sel }}} \delta P^{\mathrm{pl}, \mathrm{es}+\mathrm{bc}}\left(i_{c}(e), \overline{\overline{\epsilon^{T}}}(e)\right)\right]^{2},
$$

where $w_{P}$ is a weight that balances the displacement and pressure terms in the objective function. We chose $w_{P}=$ $1.5 N^{\text {out }} \times 10^{4} \AA^{2}$ to keep $f_{\text {min }}$ of the same order as in the previous section with $w_{P}=0$ and to reach an accuracy better
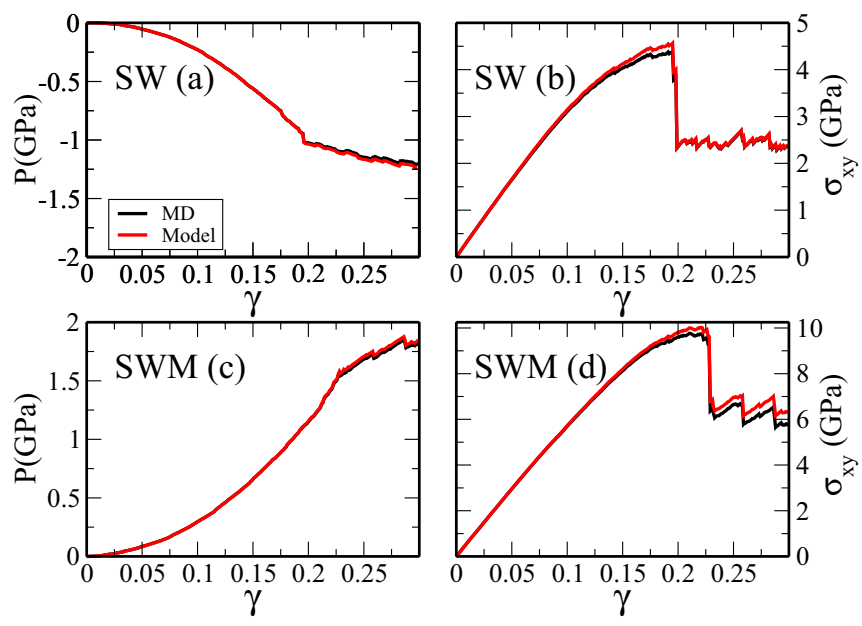

FIG. 13. Model results (red curves) including variable inclusion radius, strain-dependent elastic constants, and pressure variations in the objective function of Eq. (19), compared to MD (black curves). (a) Pressure (SW sample); (b) $\sigma_{x y}$ (SW sample); (c) pressure (SWM sample); (d) $\sigma_{x y}$ (SWM sample).

than $0.1 \mathrm{MPa}$ in the plastic pressure variations. As shown in Fig. 13, the fitting procedure then leads to a remarkable agreement between the model and the atomistic results. The fact that the model pressure depends on the choice of objective function, while the model shear stress does not, highlights the second-order nature of the displacements responsible for the hydrostatic part of the Eshelby tensors.

We see in Fig. 12 that the pressure decreases with the SW potential during plastic events, while it increases with the SWM potential. This is especially clear in the first part of the stress-strain curves, before the first shear band forms, a section well reproduced by the model, independently of the fitting technique. This observation can be linked to the typical atomistic rearrangements observed in the core of the plastic events with both potentials. As shown by the representative examples in Fig. 14, the negative volume variation with the SW potential is mainly associated with the formation of atomic bonds, leading to an increase in the atomic coordination number upon plasticity. In the SWM samples, the positive local volume variation is associated with bond disruption and formation of undercoordinated defects close to the center of the plastic events. This behavior can be rationalized by the different nature of the atomic interactions modeled by these potentials. The relatively low amplitude of the three-body parameter in the original SW potential leads to a moderate energy penalty for structures with angles that depart from the tetraheron angle of $109.47^{\circ}$, allowing for the formation of fivefold coordinated atoms upon plastic rearrangements. On the other hand, the large three-body parameter in the SWM potential gives a higher energetic penalty for angles away from $109.47^{\circ}$, making bond breaking competitive. These combined variations of volume and coordination number during plastic rearrangements are also consistent with the the ductile versus fragile behaviors observed with these potentials [30,42].

Our results show that the pressure variations are determined by the specificities of the interatomic potential, leading to an average local contraction or dilatation with the SW and SWM 

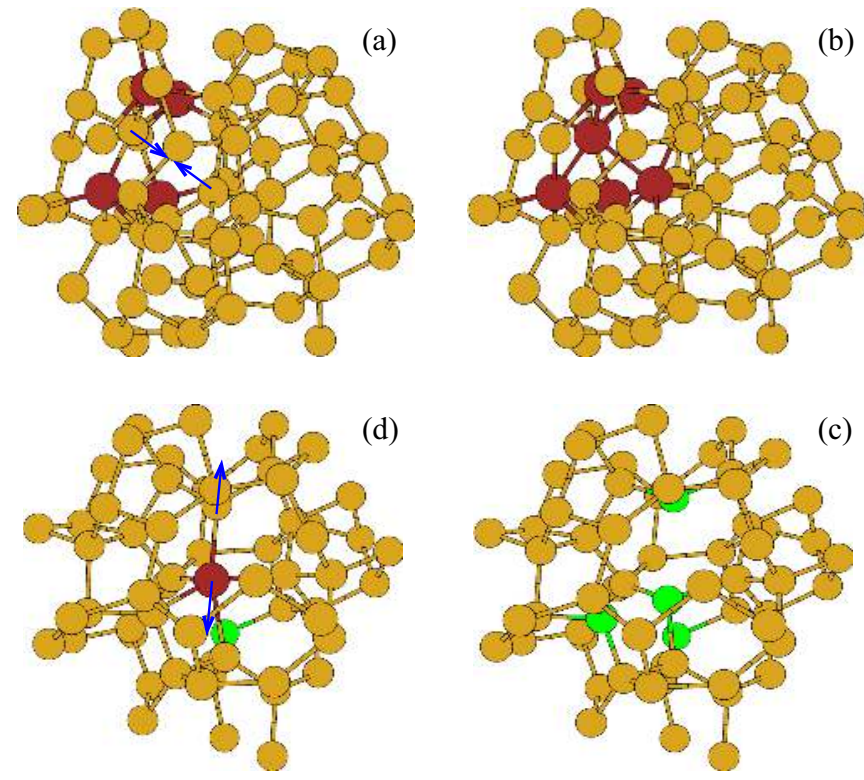

FIG. 14. Typical plastic events in an SW (a, b) and an SWM (c, d) sample. (a) SW sample: local current configuration before rearrangement; red atoms are fivefold coordinated and arrows indicate the main displacements. (b) SW sample: reversed configuration after rearrangement; new bonds are formed and the number of fivefold coordinated atoms has increased. (c, d) Same for an SWM sample, where the number of threefold coordinated atoms (green spheres) increases.

potentials, respectively. These results can be put into perspective with STZ mesoscopic models [16,43], which explicitly use a dilatancy parameter, which measures the amount of free volume generated during plastic rearrangements. In most cases, dilatancy is taken positive with reference to experiments on colloids or metallic glasses. However, this result is not general since other systems such as silica are known to exhibit permanent densification upon plasticity [39-41]. Our results indeed suggest that volume variations upon plasticity depend on the detailed interactions between particles.

\section{CONCLUSIONS}

In this paper we have presented an automated technique to extract Eshelby inclusions associated with continuum mechanics from series of atomistic configurations. A systematic analysis of the plastic energy and displacement fields allows us to efficiently select the relevant inclusions, from which the main stress-strain relations can be reproduced with fidelity. Our results confirm the central role of these inclusions in the mechanical properties of amorphous systems and therefore fully support the mesoscopic models that use them as the elementary bricks of plasticity. From a practical point of view, we have identified the main parameters that should be considered to reach a quantitative description of athermal plasticity in amorphous systems. In particular, we have shown that in silicon, the mechanical response does not depend on the volume $V_{\text {esh }}$ and transformation strain $\epsilon_{x y}^{T}$ of the Eshelby inclusions independently but, rather, depends on the product $\epsilon_{x y}^{T} V_{\text {esh }}$. We have also shown that accounting for the complex variation of the shear modulus with deformation instead of using a constant shear modulus is important in order to reproduce the stress-strain curve. Finally, we have shown that the pressure variations are more difficult to capture and depend significantly on the details of the interatomic potential.

We should note that these features were extracted in the case of a simple shear deformation. Applying a compressive or a tensile deformation may affect the relative roles played by the shear and pressure terms of the transformation strain tensor.

The main perspective of this work is to use the atomic-scale parameters obtained here to inform a mesocopic model of amorphous plasticity. This will contribute to reducing the empirical assumptions present in this type of model to achieve a better quantitative description. In this paper, only quasistatic simulations have been performed. They correspond to the very low strain rate limit of overdamped systems [25] and show that in this case plastic deformation and constitutive laws can be described as a succession of localized rearrangements that can be considered elementary bricks for plastic flow. The temporal succession of such rearrangement has not been discussed here: neither the effect of possibly competing relaxation times [44] nor the role of inertia on shear band formation [45]. Such effects are related to the nucleation and damping of the elementary rearrangements as a function of the strain rate and become important only for complex systems submitted to sufficiently high strain rates. Our work consolidates the lowtemperature and low-strain-rate limit on which larger-scale models should be based. Applications of interest will include shear localization and indentation or mechanical properties of nanosystems like nanowires.

\section{ACKNOWLEDGMENTS}

T.A. and A.T. acknowledge support from the ANR Mecasil (French Grant No. ANR-12-BS04-0004). F.B. and D.R. acknowledge support from LABEX iMUST (Grant No. ANR10-LABX-0064) of Université de Lyon (program Investissements d'Avenir; Grant No. ANR-11-IDEX-0007).

\section{APPENDIX: CONVERGENCE WITH PERIODIC BOUNDARY CONDITIONS}

The fits presented throughout this paper have been done using the minimum image convention to calculate the displacements $u_{i}$ from Eq. (6). However, a correct expression should incorporate the contribution from the periodic images of the plastic events to the displacement field. We check here the accuracy of the minimum image convention by adding up to 1331 periodic images corresponding to five cell translations in each direction. Interestingly, in the test case in Fig. 4, the fit including the images gave the same transformation strain tensor as the fit using the minimum image convention. Going beyond this simple test we considered two practical configurations from an SWM sample at $\gamma=$ 0.238 and $\gamma=0.286$ with, respectively, 8 and 77 selected plastic events. The first test is representative of the average configuration found in our calculation, while the second is associated with a more difficult situation where many plastic events localize along a shear band. In each case we performed Eshelby fits with a maximum number of $n$ cell translations 

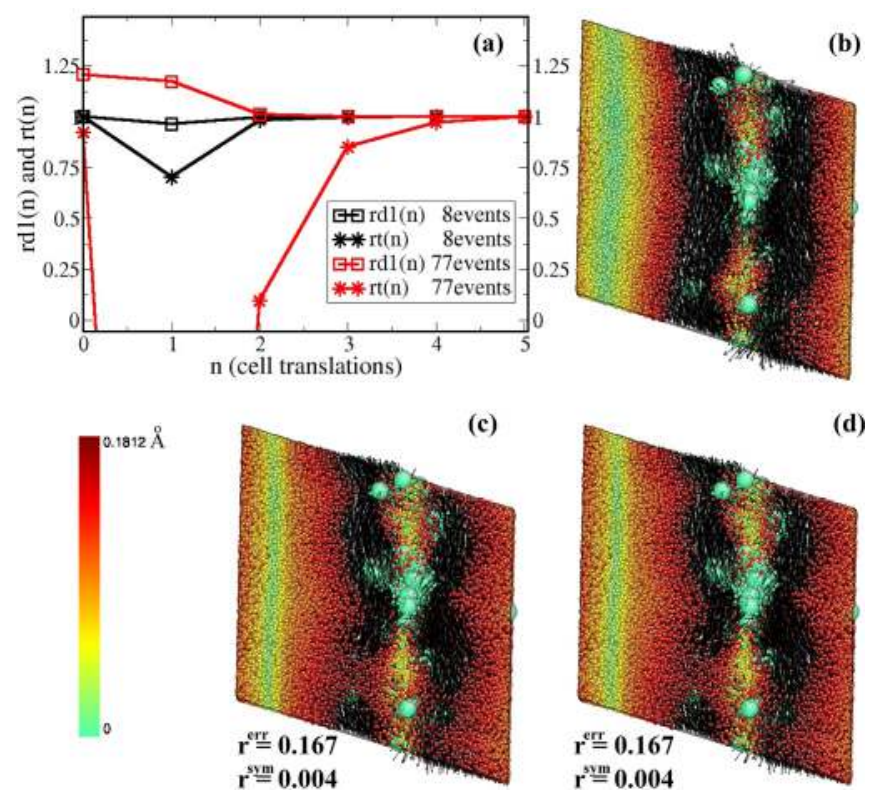

FIG. 15. (a) Convergence of the fit results with respect to the number of periodic images of the unit cell included to calculate the displacement field. $n$ is the maximum number of unit cell translations in each direction; $n=0$ corresponds to the minimum image convention. $r d 1(n)$ (squares) and $r t(n)$ (asterisks) are characteristic ratios extracted from the deviatoric and hydrostatic parts of the average transformation strain matrix $\overline{\overline{\epsilon^{\mathrm{av}}}}(n)$ (see text). Black curves: from displacement fits on an SWM configuration at $\gamma=0.238$ with eight plastic events. Red curves: from displacement fits on an SWM configuration at $\gamma=0.286$ with 77 plastic events. (b) Atomic displacements between the reversed and the initial configurations in the SWM configuration at $\gamma=0.286$. The color code refers to the modulus of the displacement, in $\AA$ (atoms with larger displacements are not shown); the arrows show the displacements; and the plastic event centers are represented by the large spheres. (c) Displacement fit using 77 Eshelby inclusions and 1331 images of the unit cell $(n=5)$. (d) Same as (c), but using the minimum image convention approximation.

in a given direction, leading to $(2 n+1)^{3}$ images for each $n$ value between $1 \leqslant n \leqslant 5$. In the output of the fit we analyze the average transformation strain tensor calculated as $\overline{\overline{\epsilon^{T \text { av }}}}(n)=\sum_{e=1}^{N_{\text {sel }}} \frac{V_{\text {esh }}(e)}{V} \overline{\overline{\epsilon^{T}}}(e, n)$. In Fig. 15(a) we plot $r d 1(n)$, the ratio of the lowest eigenvalue of the deviatoric part of $\overline{\overline{\epsilon^{T a v}}}(n)$ with respect to the same eigenvalue calculated for the better-converged reference at $n=5$, and the $r t(n)$ ratio, which is built similarly using the trace of $\overline{\overline{\epsilon^{T a v}}}$. We first observe that these ratios converge quickly as a function of $n$. In all cases, only small differences are found between $n=4$ and $n=5$. As we have found in the previous simple test, the average Eshelby strain tensors is only weakly affected by the number of periodic images in the configuration with 8 plastic events (black curves, $\gamma=0.236$ ); notably, the minimum image convention fit matches the best converged result better than the 27-image case with $n=1$. For the difficult test $(\gamma=0.286$; red curves in Fig. 15) convergence is slower as a function of $n$ and a discrepancy of about $+20 \%$ in the deviatoric component is found between the minimum image convention and the best converged result at $n=5$. This can be understood by considering that, close to the band, many events participate in each atomic displacement, and within the minimum image convention this number of contributing events is limited by the size of the cell. Consequently, fewer events in the minimum image convention induce larger strain tensor components, consistent with the above-mentioned overestimation. This error represents a good estimate of the maximum error in the shear components found in few difficult configurations over the whole strain range. We also note that the trace of $\overline{\overline{\epsilon^{T \text { av }}}}$ converges more slowly than the deviatoric part, while large variations are seen as a function of $n$ in the difficult test at $\gamma=0.286$.

Figures 15(b)-15(d) give a qualitative picture of the difficult test at $\gamma=0.286$. Despite some variations in the Eshelby strain tensors, the displacement fields produced by the minimum image convention [Fig. 15(d)] and the fit including 1331 images [Fig. 15(c)] are very similar, with almost the same values of $r^{\text {err }}$ and $r^{\mathrm{sym}}$. Their common differences from the atomistic field represented in Fig. 15(b) are located in the neighborhood of the inclusions where the displacements can still carry a strong plastic character.

These tests show that the minimum image convention applied to systems with a linear dimension of $\simeq 8.7 \mathrm{~nm}$ provides an excellent approximation of the displacement field in periodic bulk systems for most of the configurations. This approximation is expected to be less accurate when a high density of intense plastic events appears during the formation or the activation of a shear band, which represents roughly 10 difficult configurations per sample in the interval $0<\gamma<0.3$. In these few difficult cases, the minimum image configuration tends to overestimate the shear components of the transformation strain tensor. Independently of the configuration, the minimum image convention approximation will systematically be more accurate upon increasing the system size. Given its formal simplicity and its rather good accuracy we have used the minimum image convention to calculate the displacements throughout this paper.
[1] J. D. Eshelby, Proc. R. Soc. A 241, 376 (1957).

[2] G. Picard, A. Adjari, F. Lequeux, and L. Bocquet, Eur. Phys. J. E 15, 371 (2004).

[3] D. Rodney, A. Tanguy, and D. Vandembroucq, Model. Simul. Mater. Sci. Eng. 19, 083001 (2011).
[4] V. V. Bulatov and A. S. Argon, Model. Simul. Mater. Sci. Eng. 2, 167 (1994).

[5] K. Martens, L. Bocquet, and J.-L. Barrat, Phys. Rev. Lett. 106, 156001 (2011).

[6] M. Talamali, V. Petäjä, D. Vandembroucq, and S. Roux, Phys. Rev. E 84, 016115 (2011). 
[7] E. R. Homer, D. Rodney, and C. A. Schuh, Phys. Rev. B 81, 064204 (2010).

[8] R. Dasgupta, H. G. E. Hentschel, and I. Procaccia, Phys. Rev. Lett. 109, 255502 (2012).

[9] A. Nicolas, F. Puosi, H. Mizuno, and J.-L. Barrat, J. Mech. Phys. Sol. 78, 333 (2015).

[10] E. A. Jagla, Phys. Rev. E 76, 046119 (2007).

[11] A. Tanguy, F. Leonforte, and J.-L. Barrat, Eur. Phys. J. E 20, 355 (2006).

[12] A. S. Argon, Acta Metall. 27, 47 (1979).

[13] F. Spaepen, Acta Metall. 25, 407 (1977).

[14] P. Sollich, Phys. Rev. E 58, 738 (1998).

[15] M. L. Falk and J. S. Langer, Phys. Rev. E 57, 7192 (1998).

[16] J. S. Langer and L. Pechenik, Phys. Rev. E 68, 061507 (2003).

[17] V. Chikkadi, G. Wegdam, D. Bonn, B. Nienhuis, and P. Schall, Phys. Rev. Lett. 107, 198303 (2011).

[18] K. Jensen, Ph.D. thesis, Harvard University, 2013.

[19] C. Maloney and A. Lemaitre, Phys. Rev. Lett. 93, 016001 (2004).

[20] C. E. Maloney and A. Lemaître, Phys. Rev. E 74, 016118 (2006).

[21] A. Lemaître, Phys. Rev. Lett. 113, 245702 (2014).

[22] J. Ashwin, O. Gendelman, I. Procaccia, and C. Shor, Phys. Rev. E 88, 022310 (2013).

[23] D. Rodney and C. A. Schuh, Phys. Rev. Lett. 102, 235503 (2009).

[24] P. Marmottant and F. Graner, Eur. Phys. J. E 23, 337 (2007).

[25] C. Fusco, T. Albaret, and A. Tanguy, Eur. Phys. J. E 37, 43 (2014).

[26] M. Tsamados, A. Tanguy, C. Goldenberg, and J.-L. Barrat, Phys. Rev. E 80, 026112 (2009).

[27] C. Fusco, T. Albaret, and A. Tanguy, Phys. Rev. E 82, 066116 (2010).
[28] J. Tersoff, Phys. Rev. B 38, 9902 (1988).

[29] F. H. Stillinger and T. A. Weber, Phys. Rev. B 31, 5262 (1985).

[30] D. Holland and M. Marder, Phys. Rev. Lett. 80, 746 (1998); 81, 4029 (1998).

[31] K. Laaziri, S. Kycia, S. Roorda, M. Chicoine, J. L. Robertson, J. Wang, and S. C. Moss, Phys. Rev. B 60, 13520 (1999).

[32] J. Fortner and J. S. Lannin, Phys. Rev. B 39, 5527 (1989).

[33] L. B. Freund and S. Suresh, Thin Film Materials (Cambridge University Press, Cambridge, UK, 2003), p. 104.

[34] C. L. Allred, X. Yuan, M. Z. Bazant, and L. W. Hobbs, Phys. Rev. B 70, 134113 (2004).

[35] C. Goldenberg and I. Goldhirsch, Granul. Matter 6, 87 (2004).

[36] R. F. W. Bader, Chem. Rev. 91, 893 (1991).

[37] J. H. Irving and J. G. Kirkwood, J. Chem. Phys. 18, 817 (1950).

[38] A. F. Bower, Applied Mechanics of Solids (CRC Press, Boca Raton, FL, 2010).

[39] J. D. Mackenzie, J. Am. Ceram. Soc. 46, 461 (1963).

[40] T. M. Gross and M. Tomozawa, J. Appl. Phys. 104, 063529 (2008).

[41] C. Hermansen, J. Matsuoka, S. Yoshida, H. Yamazaki, Y. Kato, and Y. Z. Yue, J. Non-Cryst. Solids 364, 40 (2013).

[42] L. Pizzagalli, J. Godet, J. Guénolé, S. Brochard, E. Holmstrom, K. Nordlund, and T. Albaret, J. Phys.: Condens. Matter 25, 055801 (2013).

[43] M. Q. Jiang G. Wilde and L. H. Dai, Mech. Mater. 81, 72 (2015).

[44] T. Divoux, M.-A. Fardin, S. Manneville, and S. Lerouge, Annu. Rev. Fluid Mech. 48, 81 (2016); D. Bonn, J. Paredes, M. M. Denn, L. Berthier, T. Divoux, and S. Manneville, arXiv:1502.05281.

[45] A. Nicolas, J.-L. Barrat, and J. Rottler, Phys. Rev. Lett. 116, 058303 (2016). 